\title{
Early toxicities of ultrahypofractionated stereotactic body radiotherapy for intermediate risk localized prostate cancer using cone-beam computed tomography and real-time three- dimensional transperineal ultrasound monitoring
}

\author{
Eric Ka-Chai Lee ${ }^{1}$, Ronnie Wing-Kin Leung ${ }^{2}$, Hollis Siu-Leung Luk ${ }^{1}$, Barry Bar-Wai Wo ${ }^{1}$ \\ ${ }^{1}$ Department of Clinical Oncology, Tuen Mun Hospital, Hong Kong \\ ${ }^{2}$ Auckland Radiation Oncology, Auckland, New Zealand
}

Received: November 19, 2020

Revised: June 16, 2021

Accepted: June 17, 2021

Correspondence:

Eric Ka-Chai Lee

Department of Clinical Oncology,

Tuen Mun Hospital, 23 Tsing Chung

Koon Road, Tuen Mun, Hong Kong.

Tel: +85296249784

E-mail: leekachai2000@yahoo.com.

hk

ORCID:

https://orcid.org/0000-0003-2647-0775
Purpose: Image-guided radiotherapy (IGRT) is central to the safe and effective delivery of ultrahypofractionated (UF) stereotactic body radiotherapy (SBRT) for localized prostate cancer. We aim to study the safety of performing UF-SBRT using cone-beam computed tomography (CBCT) and real-time transperineal ultrasound (TPUS) monitoring.

Materials and Methods: We retrospectively review the medical records of 26 patients who had received UF-SBRT for intermediate risk localized prostate cancer in our institution. All patients were treated with SBRT 35-40 Gy to the clinical target volume in 5 fractions over 2-5 weeks. CBCT was used to correct for interfraction displacement while intrafraction displacement of the prostate gland was monitored using TPUS. The primary endpoints were incidence of acute toxicities and patient reported urinary toxicities in terms of the International Prostate Symptom Score: before (IPSS1), at the completion of (IPSS2), and at 3-6 months (IPSS3) after SBRT.

Results: All men were followed up for at least 3 months after SBRT. Patients experienced transient worsening of their urinary symptoms at the end of SBRT but they usually recovered in 3-6 months afterwards. The median IPSS1, IPSS2, and IPSS3 were 12, 12.5, and 8, respectively. One patient developed grade 3 rectal bleeding which was related to underlying hemorrhoid. No other grade 3-4 acute toxicity was observed.

Conclusion: It appears safe to deliver UF-SBRT without fiducial marker for prostate cancer patients using CBCT and non-invasive hybrid imaging modalities for positioning and tracking. Longer follow-up is necessary to monitor the treatment efficacy and long-term toxicities.

Keywords: Prostate cancer, Radiation, Stereotactic, Toxicities

\section{Introduction}

External radiotherapy with conventional dose-fractionation (2 Gy per fraction) is a standard definitive treatment for men with localized prostate cancer. However, the radiobiology of prostate cancer is characterized by its higher sensitivity to hypofractionation (daily delivery with fraction sizes more than 2 Gy) with low alpha/beta value being reported irregardless of the risk groups and the use of androgen deprivation. The American Society for Radiation Oncology (ASTRO) convened a task force and a strong consensus was reached for offering moderate hypofractionation across risk groups to prostate cancer patients choosing external beam radiation therapy [1]. Ultrahypofractionated (UF) radiation (> 5 Gy per fraction) may also be offered for low- and intermediate-risk prostate cancer. 
With any hypofractionated regimen, the task force strongly recommends image-guided radiation therapy (IGRT) and avoidance of non-modulated three-dimensional conformal techniques.

Stereotactic body radiotherapy (SBRT) is a novel radiotherapy technique which involves more precise planning and treatment delivery especially when UF radiation is being used. IGRT is central to the safe and effective delivery of UF-SBRT for localized prostate cancer, since undue radiation injury to the adjacent organs such as rectum and bladder will cause much detriment in patients' quality of life. An ESTRO (European Society for Radiotherapy and Oncology) panel has reached some consensus in the recommended IGRT solutions but the committee encourages further improvements to clinical practice by individual center [2]. The optimal IGRT solution remains uncertain as most SBRT studies on prostate cancer are non-randomized and some may have follow-up time that are still too short to reflect the tumor control rate and late toxicities. Nevertheless, it is prudent for researchers to monitor the acute toxicities with such novel techniques.

Most of the UF-SBRT studies for prostate cancer utilized gold seed or Calypso for positioning and tracking. However, such method requires invasive surgical procedures and may potentially be associated with markers' migration. The introduction of cone-beam computed tomography (CBCT) and real-time three-dimensional (3D) transperineal ultrasound (TPUS) Clarity system (Elekta, Stockholm, Sweden) to our institution offer a non-invasive approach for our prostate cancer patients and its efficiency and accuracy were extensively validated by many groups. Since October 2018, consecutive patients with intermediate risk localized prostate cancer were treated with UF-SBRT in our centre and the acute toxicities of the first 26 patients are reported in this study.

\section{Materials and Methods}

\section{Patient selection}

Patients with biopsy proven adenocarcinoma of prostate with staging magnetic resonance imaging (MRI) performed were referred to the combined uro-oncology clinic and selected for UF-SBRT if they fulfilled all the following criteria: (1) biopsy proven adenocarcinoma of the prostate with the International Society of Urological Pathology (ISUP) grade 3 or less; (2) prostate-specific antigen (PSA) 10-20 $\mathrm{ng} / \mathrm{mL}$; (3) $\leq \mathrm{T} 2 \mathrm{c}$ on staging MRl; (4) prostate gland volume $90 \mathrm{~mL}$ or less. Informed consent were obtained from the patients. Approval was obtained from the ethics committee of the New Territories West Cluster, Hong Kong Hospital Authority (No. NTWC/REC/20113).

\section{Simulation, planning and treatment}

Patients were given laxatives and asked to empty their rectum us- ing Dulcolax or fleet enema before they were simulated and treated in a supine position with full bladder. Dietary advice with preparatory guidelines was issued to patient on the date of consent/ moulding (Supplement A). The patients were immobilized in a customized A-cradle embedded in an in-house designed total body frame (Fig. 1A) with knee rest. TPUS was applied to the patients under lithotomy position (Fig. 1B). A bladder protocol was followed, involving voiding of urine followed by intake of $500 \mathrm{~mL}$ water and simulation or treatment after 30-45 minutes. No rectal balloon or spacer was used. In some patients who have calcifications inside their prostate glands which could be visualized on TPUS, these were served as additional markers for treatment position verification. Implanted fiducials were not used.

A planning CT scan with $3 \mathrm{~mm}$ slice thickness was performed for each patient and the clinical target volume (CTV) was contoured to include the tumor and entire prostate gland. Part of the seminal vesicles might also be included in the CTV at the discretion of the oncologist. A planning target volume (PTV) was generated with 4 $\mathrm{mm}$ margin around the CTV isotropically. Targets and organs-atrisk including rectum, bladder, bilateral femoral heads and penile bulb were delineated on the planning CT scan images using Monaco Sim (Elekta) with pre-treatment T2-weighted magnetic resonance images as reference (Fig. 1C).

A 3D ultrasound image acquired by the TPUS at the time of CT scan was registered with the planning CT with the same frame-of-reference. Position reference volume which could be the prostate or calcification was contoured in Clarity workstation for real-time intrafractional monitoring and finally approved by the oncologist (Fig. 1D).

All SBRT were planned using volumetric modulated arc therapy (VMAT) using RayStation (version 8B; Raysearch Medical Laboratories $A B$, Stockholm, Sweden). The prescribed dose was 35-40 Gy to CTV in 5 fractions, once or twice per week. The dose constraints used and criteria for major and minor deviations were shown in Table 1. The default treatment unit was an Elekta XVI Synergy $\mathrm{S}$ model with Agility $5 \mathrm{~mm}$ multileaf collimator, 6-MV photon beam (dose rate, 1,400 MU/min) with flattening-filter-free (FFF) modification. The treatment was being delivered with either a single full $\operatorname{Arc}\left(360^{\circ}\right.$ with 91 segments, delivery time around 85 seconds, approximately 1,600 MU) or a pair of partial Arc (160 > 200 then $200>160$ with 162 segments: delivery time around 130 seconds, approximately 2,100 MU) depending on the complexity.

Image guidance was carried out at each fraction with kilovoltage (kV) CBCT on a HexaPod couch which is capable of 6D correction. The treating radiographers were trained to identify the prostate, rectum, and bladder on volumetric images. The same clinical oncologist who contoured the targets on planning CT was present to 

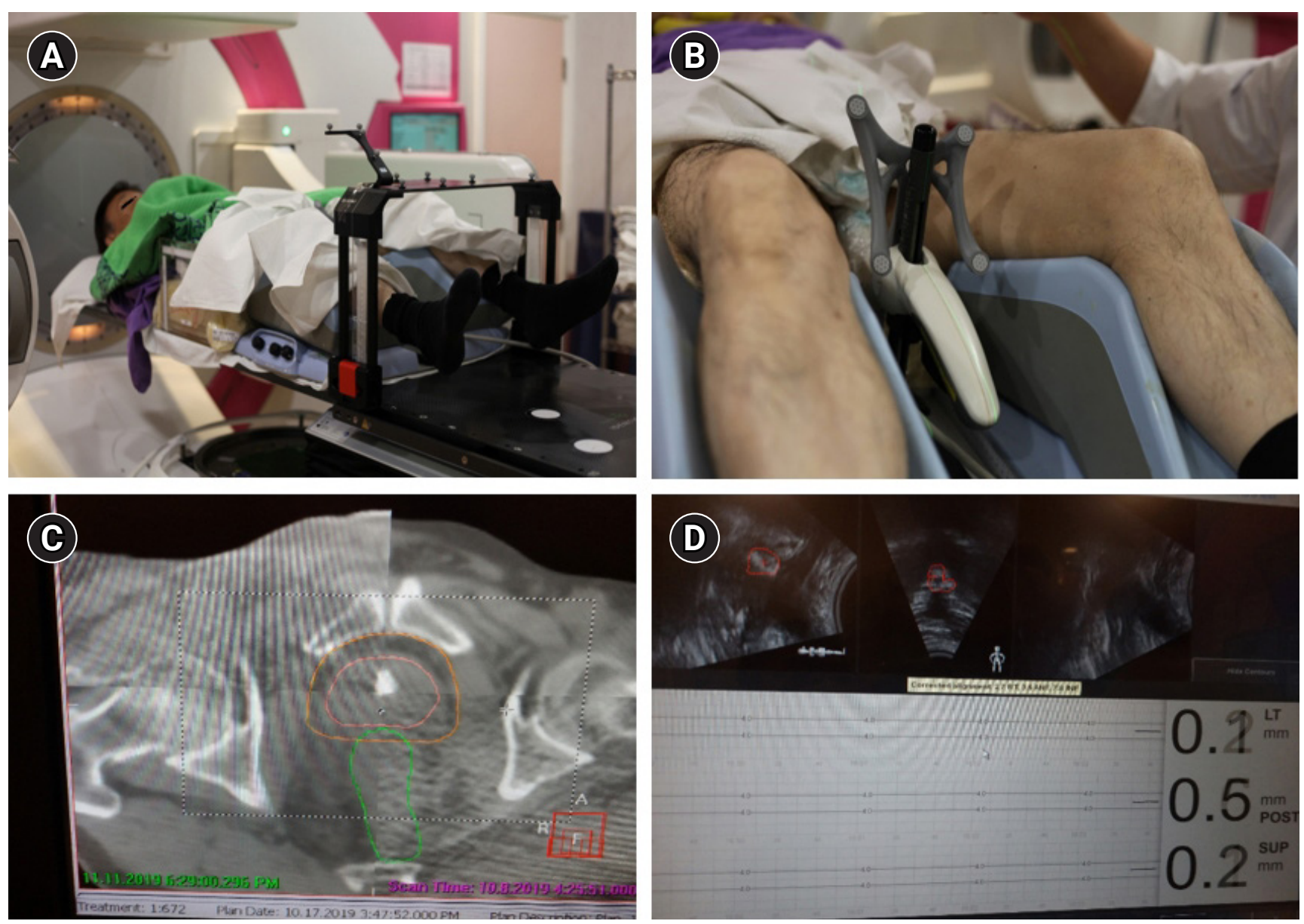

Fig. 1. (A) Total body frame. (B) Transperineal ultrasound probe. (C) Planning and cone-beam computed tomography co-registration with prostatic calcification as "fiducial marker." (D) Real-time intrafractional monitoring by transperineal ultrasound.

make the final decision on the imaging review before the delivery of each fraction. If the presence of rectal distension on the pre-treatment CBCT led to a suboptimal match, the treatment was not delivered and the patient was taken off the couch and asked to pass gas/stool. Following this the image guidance was repeated before treatment delivery.

While treatment was being delivered, a medical physicist was present to track the real-time prostate movement using the TPUS with $4 \mathrm{~mm}$ isotropic warning level. Radiation is halted if persistent prostatic displacement ( $>5$ seconds) is exceeding the warning level. Following treatment delivery, another CBCT scan was carried out to document the intrafractional anatomic change. Image guidance was always focused on the prostate, with rotational deviation less than $0.5^{\circ}$ and translational shift less than $2 \mathrm{~mm}$ on CBCT image. Minor adjustment was allowed due to rectal and bladder fillings.

In accordance with the National Comprehensive Cancer Network guideline for androgen deprivation therapy (ADT) for patients with intermediate risk localized prostate cancer, all patients also received neoadjuvant ADT with Luteinizing hormone-releasing hor- mone agonist/antagonist for 3 months and concurrent/adjuvant ADT for a total of 6 months.

\section{Patient follow-up and evaluation}

All patients were evaluated by a clinical oncologist throughout the treatment course including at baseline visit, during neoadjuvant ADT, before SBRT, at the end of and 3 months after SBRT. Serial PSA measurements were taken at each visit. Patient reported outcomes were captured by asking patients to report their urinary symptoms using the IPSS. Besides, we also reviewed the subjects' electronic patient record which included the consultation notes composed by their family physicians and other specialists. All adverse events during and within 90 days of the completion of SBRT were recorded and graded according to the Common Terminology Criteria for Adverse Event (CTCAE) v5.0.

\section{Statistical analysis}

The primary objective of this study is a descriptive analysis of the acute toxicities experienced by patients during and within 90 days 
Table 1. Dose constraints

\begin{tabular}{|c|c|c|}
\hline \multirow{2}{*}{ OAR } & \multicolumn{2}{|c|}{ Dose constraints } \\
\hline & $35 \mathrm{~Gy}$ in $5 \mathrm{fx}$ & $40 \mathrm{~Gy}$ in $5 \mathrm{fx}$ \\
\hline CTV & $V_{35 G y} \geq 99 \%$ & $\mathrm{~V}_{40 \mathrm{~Gy}} \geq 99 \%$ \\
\hline \multirow[t]{4}{*}{ PTV } & $V_{33.25 G y} \geq 99 \%$ & $\mathrm{~V} 3_{6.25 G y}>99 \%$ \\
\hline & & (accept $>95 \%$ provide $\mathrm{V}_{33.25 \mathrm{y}}>99 \%$ ) \\
\hline & $\mathrm{V}_{36.756 y}<5 \%$ & $\mathrm{~V}_{42 \mathrm{~Gy}}<5 \%$ \\
\hline & $V_{38.5 G y}<1 \%$ & $V_{44 G y}<1 \%$ \\
\hline \multirow[t]{5}{*}{ Rectum } & $\mathrm{V}_{18.1 \mathrm{~Gy}}<40 \%$ & $V_{18.1 G y}<40 \%$ \\
\hline & $V_{28 G y} \leq 15 \%$ (ideally $<12 \%$ ) & $V_{28 G y} \leq 15 \%$ (ideally $<12 \%$ ) \\
\hline & $\mathrm{V}_{35 \mathrm{~Gy}}<1 \mathrm{~mL}$ & $\mathrm{~V}_{38 G \mathrm{y}}<1 \mathrm{~mL}$ \\
\hline & & $\mathrm{V}_{356 \mathrm{y}}<2 \mathrm{~mL}$ (up to $<4 \mathrm{~mL}$ ) \\
\hline & $\mathrm{D}_{\max }=37.5 \mathrm{~Gy}$ & $\mathrm{D}_{\max }<40.6 \mathrm{~Gy}$ \\
\hline \multirow[t]{4}{*}{ Bladder } & $\mathrm{V}_{18.1 \mathrm{~Gy}}<40 \%$ & $\mathrm{~V}_{18.1 \mathrm{~Gy}}<40 \%$ \\
\hline & $V_{28 G y} \leq 15 \%$ & $V_{28 G y} \leq 15 \%$ \\
\hline & $V_{35 G y}<5 \%$ & $\mathrm{~V}_{38 G y}<6 \mathrm{~mL}$ \\
\hline & $\mathrm{D}_{\max }=37.5 \mathrm{~Gy}$ & $\mathrm{~V}_{39.56 \mathrm{y}}<2 \mathrm{~mL}$ \\
\hline \multirow[t]{2}{*}{ Femurs } & $V_{17.56 y}<15 \%$ & $V_{17.56 y}<5 \%$ \\
\hline & $\mathrm{V}_{30 \mathrm{~Gy}}<10 \mathrm{~mL}^{\mathrm{a})}$ & $\mathrm{V}_{30 \mathrm{~Gy}}<2 \mathrm{~mL}$ \\
\hline \multirow[t]{4}{*}{ Small and large bowels } & $\mathrm{V}_{25 G y}<5 \mathrm{~mL}$ & $\mathrm{~V}_{25 \mathrm{~Gy}}<5 \mathrm{~mL}$ \\
\hline & $\mathrm{V}_{30 G \mathrm{y}}<2 \mathrm{~mL}$ & $\mathrm{~V}_{30 \mathrm{~Gy}}<2 \mathrm{~mL}$ \\
\hline & $\mathrm{D}_{\max }=38 \mathrm{~Gy}$ & $\mathrm{D}_{\max }<38 \mathrm{~Gy}^{\mathrm{a})}$ \\
\hline & & (up to $D_{\max }<40$ Gy large bowel only) \\
\hline \multirow[t]{2}{*}{ Penile bulb } & $V_{35 G y}<4 \%$ & $\mathrm{~V}_{356 y}<4 \%$ \\
\hline & $\mathrm{V}_{20 \mathrm{~Gy}}<40 \%$ & $\mathrm{~V}_{20 \mathrm{~Gy}}<40 \%$ \\
\hline
\end{tabular}

OAR, organ-at-risk; CTV, clinical target volume; PTV, planning target volume.

For the 35 Gy prescription dose, conformity index $(\mathrm{Cl})=\frac{3325 \mathrm{cGy} \text { isodose volume }}{\text { PTV volume }}$.

For the 40 Gy prescription dose, conformity index $(\mathrm{Cl})=\frac{3625 \mathrm{cGy} \text { isodose volume }}{\text { PTV volume }}$.

${ }^{a)}$ Critical OAR doses should be met at the expense of target coverage.

of completion of SBRT. The IPSS before, during and 3-month after SBRT were compared using analysis of variance (ANOVA) method. All statistical analyses were based on a snapshot of data taken on March 31, 2021 and conducted with IBM SPSS Statistics for Windows, version 24.0 (IBM Corp, Armonk, NY, USA). p-value less than 0.05 was considered statistically significant.

\section{Results}

\section{Patients and treatment delivery}

Twenty-six men were treated with UF-SBRT from October 30, 2018 to December 31, 2020. Their median age was 68.5 years (range, 62 to 76 years). Table 2 illustrated their tumor characteristics. There was one patient whose presenting PSA was $71.9 \mu \mathrm{g} / \mathrm{L}$. He had a prostate-specific membrane antigen positron emission tomography (PSMA-PET) scan done which showed benign prostatic hypertrophy and tumor at transitional zone (T2cNOMO) with Gleason score $3+$
4 and he was considered to have intermediate-risk prostate cancer. The first 11 consecutive patients received 35 to CTV in 5 weekly fractions. The subsequent patients were treated to $40 \mathrm{~Gy}$ in 5 fractions delivered over 2 weeks except for one patient whose prostate had a size of $88 \mathrm{~mL}$ and he received 35 Gy to the CTV because we wanted to keep the bladder tolerance as the higher priority.

\section{Acute urinary toxicities}

As of March 15, 2021, all patients had completed UF-SBRT for at least 3 months. The median follow-up is 8.6 months (range, 3 to 28 months). Twenty-five had their IPSS recorded before (IPSS1), at the end of (IPSS2), and 3-6 months after (IPSS3) after UFSBRT. One patient had his baseline IPSS1 missing and was excluded from the analysis. The median IPSS1, IPSS2, and IPSS3 were 12, 12.5, and 8, respectively (Fig. 2). Using ANOVA, there was a statistically significant difference between the mean IPSS1 to IPSS3 and post-hoc multiple comparison showed the mean 
Table 2. Patient and tumor characteristics

\begin{tabular}{|c|c|c|c|c|}
\hline Patients\# & Age (yr) & T-stage & PSA ( $\mu \mathrm{g} / \mathrm{L})$ & Gleason score \\
\hline 1 & 70 & $2 c$ & 10.60 & $4+3$ \\
\hline 2 & 65 & $2 a$ & 13.30 & $3+3$ \\
\hline 3 & 76 & $2 c$ & 11.80 & $3+3$ \\
\hline 4 & 74 & $1 \mathrm{c}$ & 19.30 & $3+3$ \\
\hline 5 & 66 & $1 \mathrm{c}$ & 6.40 & $3+4$ \\
\hline 6 & 74 & $2 c$ & 18.10 & $3+3$ \\
\hline 7 & 60 & $2 c$ & 7.10 & $4+3$ \\
\hline 8 & 68 & $2 c$ & 13.10 & $3+4$ \\
\hline 9 & 71 & $2 c$ & 71.90 & $3+4$ \\
\hline 10 & 61 & $2 c$ & 8.95 & $4+3$ \\
\hline 11 & 73 & $2 c$ & 17.80 & $4+3$ \\
\hline 12 & 68 & $2 a$ & 11.80 & $3+4$ \\
\hline 13 & 78 & $2 b$ & 10.87 & $3+4$ \\
\hline 14 & 70 & $2 c$ & 17.10 & $3+3$ \\
\hline 15 & 62 & $2 b$ & 19.10 & $3+4$ \\
\hline 16 & 69 & $2 c$ & 14.80 & $4+3$ \\
\hline 17 & 67 & $2 a$ & 7.06 & $4+3$ \\
\hline 18 & 68 & $2 a$ & 7.20 & $4+3$ \\
\hline 19 & 75 & 3a & 18.70 & $4+3$ \\
\hline 20 & 66 & $2 b$ & 11.10 & $4+3$ \\
\hline 21 & 76 & $2 c$ & 19.10 & $3+4$ \\
\hline 22 & 69 & $2 c$ & 18.90 & $3+3$ \\
\hline 23 & 76 & $2 c$ & 18.50 & $3+3$ \\
\hline 24 & 71 & $2 c$ & 8.02 & $4+3$ \\
\hline 25 & 77 & $2 c$ & 20.00 & $3+3$ \\
\hline 26 & 72 & $1 c$ & 12.00 & $4+3$ \\
\hline
\end{tabular}

PSA, prostate-specific antigen.

IPSS2 was significantly higher than the IPSS3, respectively ( $p=$ 0.04) but no statistical significant difference was found between mean IPSS1 and IPSS3. This implied that patients generally had their urinary symptoms worsened at the end of UF-SBRT but they could recover at 3-6 months after treatment completion. Two patients noted mild hematuria which subsided spontaneously. Another patient had experienced grade 3 hematuria and he had flexible cystoscopy performed which revealed the hematuria was due to bladder stones only.

\section{Non-urinary acute toxicities}

One patient who was treated to $35 \mathrm{~Gy}$ in 5 fractions complained of outlet type rectal bleeding about 1 week after UF-SBRT and he had colonoscopy performed which revealed hemorrhoid and his symptom subsided after conservative treatment. The acute toxicities noted in other patients were of grade 1-2 only. These included erection dysfunction in four patients, anal and scrotal radiation dermatitis in five patients and vertigo in one patient. Three patients complained of diarrhea/increase passage of loose stool due to the

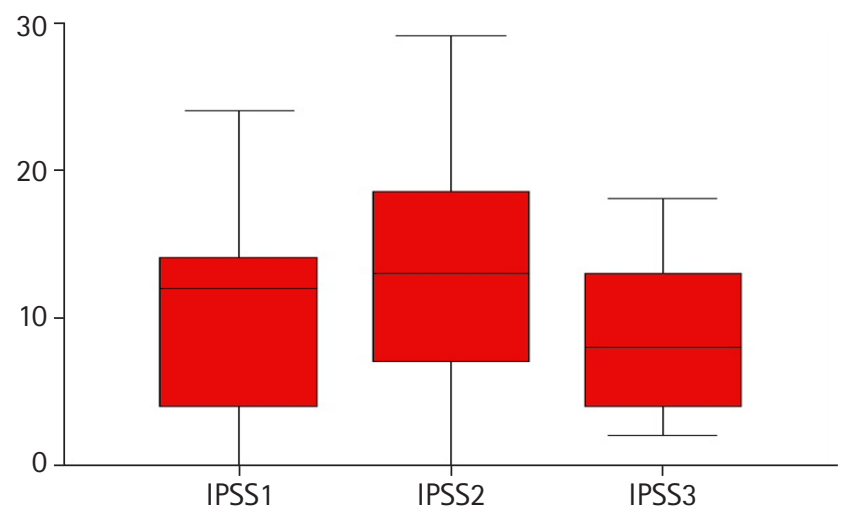

Fig. 2. Patient reported acute urinary toxicities using the International Prostate Symptom Score: before (IPSS1), at the completion of (IPSS2), and at 3-6 months (IPSS3) after stereotactic body radiotherapy.

Table 3. Number of patients with non-urinary acute toxicities according to CTCAE v5.0

\begin{tabular}{lccccc}
\hline & Grade 1 & Grade 2 & Grade 3 & Grade 4 & Grade 5 \\
\hline $\begin{array}{l}\text { Erectile dysfunction } \\
\text { Radiation dermatitis }\end{array}$ & 4 & 0 & 0 & 0 & 0 \\
$\quad \begin{array}{l}\text { over around anal and } \\
\text { scrotal area }\end{array}$ & & 2 & 0 & 0 & 0 \\
$\begin{array}{l}\text { Flatulence } \\
\text { Rectal/hemorrhoidal }\end{array}$ & 1 & 0 & 1 & 0 & 0 \\
$\quad$ bleeding & & & & & \\
Diarrhoea/loose stool & 3 & 0 & 0 & 0 & 0 \\
Rectal pain/urge & 2 & 0 & 0 & 0 & 0 \\
Constipation & 1 & 0 & 0 & 0 & 0 \\
Epigastric discomfort & 1 & 0 & 0 & 0 & 0 \\
Vertigo & 1 & 0 & 0 & 0 & 0 \\
\hline
\end{tabular}

CTCAE, Common Terminology Criteria for Adverse Event.

use of laxative during SBRT. Two patients complained of increase rectal urge/pain and two patients complained of increase flatulence (Table 3).

\section{Treatment efficacy}

The PSA of all 26 men were noted to respond to ADT and SBRT and fall below $4 \mathrm{ng} / \mathrm{mL}$ at 3-6 months after UF-SBRT (Table 4). Thirteen patients had their PSA become undetectable $(<0.03 \mu \mathrm{g} / \mathrm{L})$. The treatment efficacy (excluding the patient with initial PSA $71.9 \mu \mathrm{g} / \mathrm{L}$ ) in terms of the PSA response is depicted in Fig. 3.

\section{Discussion and Conclusion}

In 2018, the ASTRO issued a guideline which recommended the use of moderately hypofractionated radiation therapy for localized prostate cancer. There was a conditional consensus for UF radiation therapy in intermediate-risk prostate cancer. The results of two 
Table 4. Treatment efficacy (PSA response)

\begin{tabular}{|c|c|c|c|}
\hline \multirow[b]{2}{*}{ Patients\# } & \multicolumn{3}{|c|}{ PSA $(\mu \mathrm{g} / \mathrm{L})$} \\
\hline & $\begin{array}{l}\text { Before any } \\
\text { treatment }\end{array}$ & $\begin{array}{l}\text { After neoadjuvant } \\
\text { ADT/before SBRT }\end{array}$ & $\begin{array}{l}\text { 3-6 months } \\
\text { after SBRT }\end{array}$ \\
\hline 1 & 10.60 & 0.70 & 0.10 \\
\hline 2 & 13.30 & 3.14 & 0.26 \\
\hline 3 & 11.80 & 5.50 & 0.14 \\
\hline 4 & 19.30 & 5.11 & 3.68 \\
\hline 5 & 6.40 & 0.28 & $<0.03$ \\
\hline 6 & 18.10 & 0.27 & $<0.03$ \\
\hline 7 & 7.10 & 2.59 & 0.58 \\
\hline 8 & 13.10 & 0.49 & 0.03 \\
\hline 9 & 71.90 & 4.66 & $<0.03$ \\
\hline 10 & 8.95 & 0.55 & $<0.03$ \\
\hline 11 & 17.80 & 0.73 & 0.03 \\
\hline 12 & 11.80 & 3.87 & 0.67 \\
\hline 13 & 10.87 & 1.12 & $<0.03$ \\
\hline 14 & 17.10 & 0.22 & $<0.03$ \\
\hline 15 & 19.10 & 2.21 & 0.12 \\
\hline 16 & 14.80 & 0.28 & $<0.03$ \\
\hline 17 & 7.06 & 1.34 & 0.10 \\
\hline 18 & 7.20 & 0.09 & $<0.03$ \\
\hline 19 & 18.70 & 1.42 & $<0.03$ \\
\hline 20 & 11.10 & 3.84 & 0.43 \\
\hline 21 & 19.10 & 0.65 & $<0.03$ \\
\hline 22 & 18.90 & 5.82 & 0.13 \\
\hline 23 & 18.50 & 0.17 & $<0.03$ \\
\hline 24 & 8.02 & 0.94 & 0.11 \\
\hline 25 & 20.00 & 0.03 & $<0.03$ \\
\hline 26 & 12.00 & 0.25 & $<0.03$ \\
\hline
\end{tabular}

PSA, prostate-specific antigen; ADT, androgen deprivation therapy; SBRT, stereotactic body radiotherapy.

randomized studies had reported early and 5-year outcomes of UFSBRT as compared to conventional fractionated radiation therapy. While UK-PACE study [3] did not suggest UF-SBRT increase either gastrointestinal and genitourinary acute toxicity, the Hypo-RT-PC study [4] demonstrated similar failure-free survival and late toxicity between UF radiation therapy and conventionally fractionated radiotherapy for intermediate- to high-risk prostate cancer. Both these studies had advocated implantation of fiducials as an IGRT solution. Combining implanted fiducial markers with CBCT may help to reduce inter-observer variability compared to soft-tissue alignment alone. However, in our centre the same clinical oncologist was present throughout the planning and treatment process and therefore such variability could be assumed negligible.

As proposed by Grimwood et al. [5], in a prostate SBRT setting, both Clarity and CBCT can be used. In this study, we have found UF-SBRT without fiducial marker for prostate cancer patients using

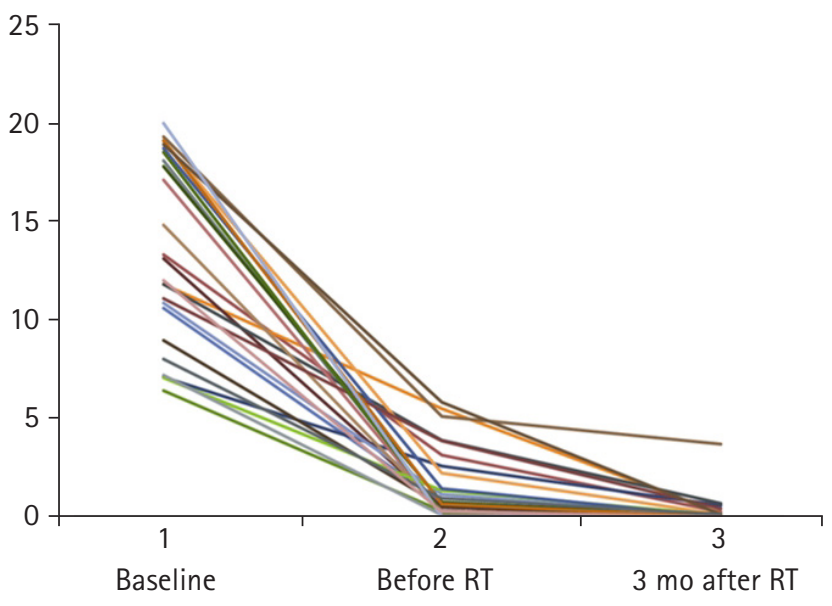

Fig. 3. Treatment efficacy (prostate-specific antigen response). RT, radiation therapy.

CBCT and non-invasive hybrid imaging modalities for positioning and tracking was safe and feasible. An online correction protocol based on soft tissue volumetric matching on CBCT images, with translational and rotational adjustment using 6 degree-of-freedom couch could be an effective adaptive strategy to control for most interfractional displacement. Besides, the Clarity TPUS Autoscan measured intrafractional prostate motion during treatment delivery. Since our CTV to PTV margin was $4 \mathrm{~mm}$, we set a gating threshold of $4 \mathrm{~mm}$ for shifts $>5$ seconds such that any intrafractional displacement above such threshold would have alarmed us to withhold treatment delivery. In fact, our previous experience with TPUS had shown the mean intrafraction prostate motions were within $4 \mathrm{~mm}$ for a typical VMAT treatment duration of 3-4 minutes [6]. In this study, the intrafractional prostate motions were usually less than $4 \mathrm{~mm}$ because each treatment time was about 3-4 minutes using FFF technique. However, the CTV to PTV margin of $4 \mathrm{~mm}$ is still needed to correct for interfractional displacement/ deformation of the prostate.

Among our treated patients, a transient worsening of their urinary symptoms was demonstrated by the longitudinal measurement of their IPSS before, during and 3-6 months after UF-SBRT. Most of these urinary toxicities were of low grade only. There had been no statistical difference in the IPSS1 and IPSS3 which implied that most patients could have recovered from the acute urinary toxicity in 3-6 months after UF-SBRT. Apart from the two patients who had underlying bowel and bladder pathology (hemorrhoid and bladder stone), there was no grade 3-4 acute radiation induced toxicities noted.

Normal tissue tolerance is also a function of the prescribed dose and treatment duration. The Sunnybrook Hospital compared the toxicities experienced by patients treated in three prospective tri- 
als: (1) 35 Gy in 5 fractions over 29 days; (2) 40 Gy in 5 fractions over 29 days; and (3) 40 Gy in 5 fractions over 11 or 29 days. The odds of late genitourinary toxicity were higher for patients treated with $40 \mathrm{~Gy}$ compared to those treated with $35 \mathrm{~Gy}$ and also higher for patients treated over 11 days as compared to 29 days [7]. However dose escalated SBRT to 40 Gy in 5 fractions was associated with lower nadir PSAs and PSA $<0.4 \mathrm{ng} / \mathrm{mL}$ was a predictor of long-term biochemical control $[8,9]$. In the PACE-B study, the SBRT PTV dose was $36.25 \mathrm{~Gy}$ in 5 fractions over 1-2 weeks (i.e., daily or alternate days, at centre discretion), with additional secondary CTV dose target of $40 \mathrm{~Gy}$. We have been treating our patients with a similar dose-fractionation regimen. In line with Brand et al. [3], the absence of increased acute toxicity from UF-SBRT is reassuring for us to continue treating patients with intermediate-risk prostate cancer using this protocol.

The limitations of our retrospective study include the small patient number from a single institution and short follow-up. We did not compare the outcomes of patients who received two dose levels (35 Gy vs. $40 \mathrm{~Gy}$ ) because the incidence of acute toxicities had remained low. Results regarding late toxicity and long term biochemical control have to be reported in the next 3-4 years.

In conclusion, our study demonstrated the safety of performing UF-SBRT for prostate cancer patients without the use of implanted fiducial markers. For patients who refuse implantation of fiducial markers or who cannot have this procedure, IGRT using CBCT and real-time TPUS may be an appropriate option.

\section{Conflict of Interest}

No potential conflict of interest relevant to this article was reported.

\section{Supplementary Materials}

Supplementary materials can be found via https://doi.org/10.3857/ roj.2020.00969.

\section{References}

1. Morgan SC, Hoffman K, Loblaw DA, et al. Hypofractionated radiation therapy for localized prostate cancer: executive summary of an ASTRO, ASCO, and AUA evidence-based guideline. Pract Radiat Oncol 2018;8:354-60.

2. Ghadjar P, Fiorino C, Munck Af Rosenschold P, Pinkawa M, Zilli T, van der Heide UA. ESTRO ACROP consensus guideline on the use of image guided radiation therapy for localized prostate cancer. Radiother Oncol 2019;141:5-13.

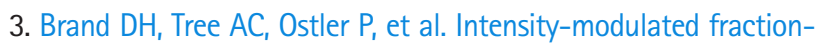
ated radiotherapy versus stereotactic body radiotherapy for prostate cancer (PACE-B): acute toxicity findings from an international, randomised, open-label, phase 3, non-inferiority trial. Lancet Oncol 2019;20:1531-43.

4. Widmark A, Gunnlaugsson A, Beckman L, et al. Ultra-hypofractionated versus conventionally fractionated radiotherapy for prostate cancer: 5-year outcomes of the HYPO-RT-PC randomised, non-inferiority, phase 3 trial. Lancet 2019;394:385-95.

5. Grimwood A, McNair HA, O'Shea TP, et al. In vivo validation of Elekta's Clarity Autoscan for ultrasound-based intrafraction motion estimation of the prostate during radiation therapy. Int J Radiat Oncol Biol Phys 2018;102:912-21.

6. Pang EP, Knight K, Fan Q, et al. Analysis of intra-fraction prostate motion and derivation of duration-dependent margins for radiotherapy using real-time 4D ultrasound. Phys Imaging Radiat Oncol 2018;5:102-7.

7. Helou J, D'Alimonte L, Quon H, et al. Stereotactic ablative radiotherapy in the treatment of low and intermediate risk prostate cancer: is there an optimal dose? Radiother Oncol 2017;123: 478-82.

8. Alayed Y, Cheung P, Pang G, et al. Dose escalation for prostate stereotactic ablative radiotherapy (SABR): late outcomes from two prospective clinical trials. Radiother Oncol 2018;127:213-8.

9. Musunuru HB, Quon H, Davidson M, et al. Dose-escalation of five-fraction SABR in prostate cancer: toxicity comparison of two prospective trials. Radiother Oncol 2016;118:112-7. 